\title{
Development of Machine Vision System for Automatic Inspection of Vehicle Identification Number
}

\author{
Yarlagadda Ramshankar, Deivanathan $\mathrm{R}^{*}$ \\ SMBS, VIT University, Chennai 600127, India
}

Received: 02 September 2017; Accepted: 08 January 2018; Published: 08 March 2018

\begin{abstract}
The vision system is developed to reduce the human effort and improve productivity in the Vehicle Quality Assurance (VQA) shop for inspection of a car bearing a Vehicle Identification Number (VIN), assigned to it at the assembly shop. This project work is carried out in association with M/s Renault Nissan Automotive India Pvt Ltd, Chennai.

The vision system consists of a camera fixed on a pan-tilt camera frame and an Optical Character Recognition (OCR) software. The camera frame is mounted on a belt conveyor with remote control of forward, backward and tilting motion. The image of VIN present at the car door is captured through a digital camera placed adjacent to the car. The characters in the VIN image thus obtained are extracted using MATLAB, with configurable OCR software. Template matching method is followed in the OCR process. The MATLAB code can overcome trivial issues in VIN image inspection at the quality shop. Development of a graphic user interface to the software is also described.
\end{abstract}

Index Terms: Vehicle identification number (VIN), machine vision, Optical Character Recognition (OCR) Arduino control camera fixture.

(C) 2018 Published by MECS Publisher. Selection and/or peer review under responsibility of the Research Association of Modern Education and Computer Science.

\section{Introduction}

An inspection system using machine vision is an advanced technology that integrates electronic equipment with software systems to perform noncontact verification of inspected features. Machine vision systems are used in different applications and much more are being developed day by day. There are two types of machine vision systems, one is computer interfaced with a camera and the other is smart camera type. Machine vision is

\footnotetext{
* Corresponding author. Tel.: +9144 39931166
}

E-mail address: deivanathan.r@vit.ac.in 
an innovative method for VIN inspection, though it needs to be performed manually sometimes. VIN is a 17 digit unique alphanumeric code, used in automotive industry to identify the individual production line vehicle. VIN is punched on the chassis and is later duplicated on stickers placed at many locations around the vehicle.

\begin{tabular}{|ll|}
\hline Nomenclature & \\
VQA & Vehicle Quality Assurance \\
VIN & Vehicle Identification Number \\
OCR & Optical Character Recognition \\
CNC & Computer Numerical Control \\
Arduino IDE & Arduino Integrated Development Environment \\
GUI & Graphic User Interface \\
CCD & Charge Coupled Device \\
USB & Universal Serial Bus \\
\hline
\end{tabular}

Presently, a human operator performs the VIN verification for vehicles entering the quality shop (after assembly) and detects any mismatch between the VIN on a vehicle and the corresponding VIN in a documented data file. Many industries in the automobile sector are adopting automated VIN inspection as a replacement for a manual process to avoid time-consuming and inconsistent results between operators. In this paper, a vision system is proposed, that has a digital camera interfaced with a computer for simple and userfriendly inspection system.

Many successful applications of vision system for license plate recognition have been reported in the automotive field. Typically, in application software designed for the number plate recognition of a vehicle, the location of number plate is first detected using morphological operation. Then, separation of the number plate characters is carried out by segmentation. Finally, the character image is normalized and template matching is applied for recognition of number plate characters $[1,2,3]$. Algorithms are used for converting the textual image of number plate into editable text. Image processing algorithms that involve multiple template matching, variable light intensity and other environmental factors are more efficient [4]. Digital Signal Processing toolbox and Computer Vision System toolbox are used for developing the SIMULINK model in MATLAB, to extract the alphabet characters on the number plate and to match the same with template images based on a pixel by pixel calculation of a metric value for the match [5]. A neural network based Optical Character Recognition (OCR) system for identification of VIN has been implemented by digitally extracting and segmenting the characters from the stamped VIN of a vehicle. For a particular vehicle, VIN images taken at different distances and angle of view are given as the input to the neural network [6, 12]. Other machine vision applications include printed character recognition on electronic components, cartridge fuse, automotive component assemblies [7-11].

\section{Overview of Proposed VIN Inspection System}

The work presented is focused on design and development of vision system for automatic identification of VIN, present in the form of a 'printed VIN sticker' on the car chassis. This needs a strategic camera location and an automatic (or) joystick control in positioning of the inspection system to capture VIN image of a vehicle arriving at the VQA shop. Subsequent to image capture, it is processed off-line by OCR program in MATLAB and displayed for verification. Fig. 1 is an overview of the proposed system for VIN identification.

\subsection{Adjustable Camera setup for Image Capture}

The major component used for the vision system is a movable camera mounted on the pan tilt stand which can slide and rotate about the horizontal and vertical axes respectively, as configured by the user, with the help of stepper motor. While the vehicle approaches the inspection system, the camera mounted on the frame should 
be adjusted to focus at the center pillar of chassis for proper image capturing, as indicated in Fig. 2, 3 and 4.

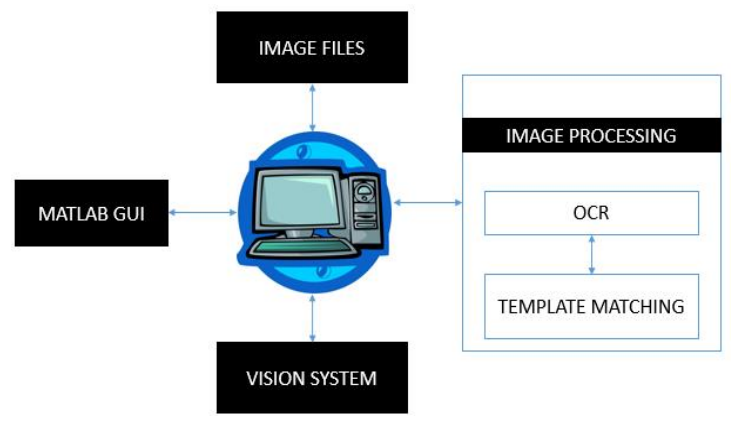

Fig.1. Overview of the system for VIN inspection.

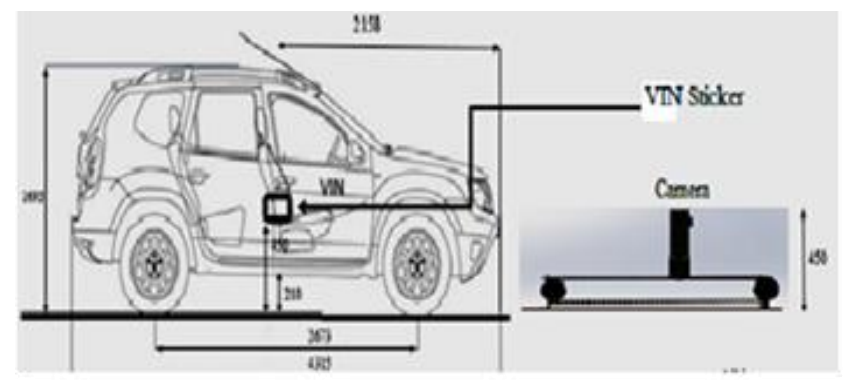

Fig.2. Side view of camera location for inspection

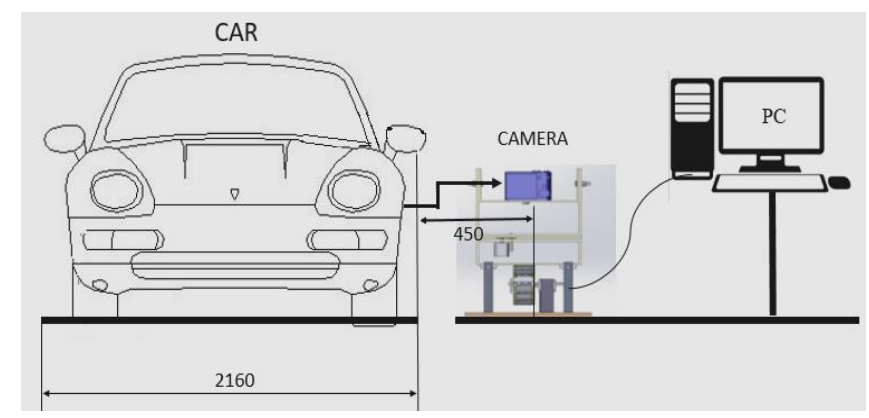

Fig.3. Front view of Camera Location for Inspection

The VIN sticker is located on the center pillar of chassis near the door and when the door is opened fully, the image will be visible on the camera display. If the visibility is poor due to positioning, minor adjustment is given to the camera position. The camera mount should be rotated with respect to the position of VIN location using a stepper motor controlled by a joystick or by giving fixed rotation step pulse to the stepper motor. By this, we can conveniently position the camera towards the target. The following discussion details the various processes involved from VIN imaging to VIN verification. 


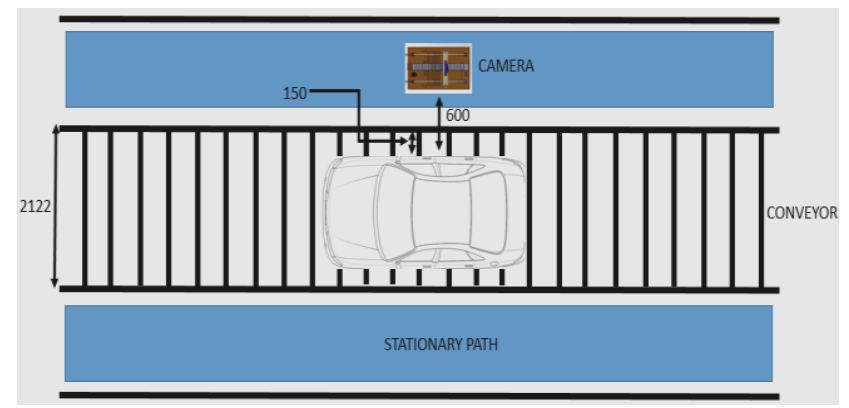

Fig.4. Top View of Camera Location For Inspection

\subsection{Optical Character Recognition(OCR) Process}

OCR technology enables us to convert the scanned paper documents, PDF files or images captured by a digital camera into editable and searchable data. The classical techniques for OCR are Template matching, Statistical and Structural methods, while the soft computing techniques such as Artificial Neural Networks (ANNs), Fuzzy logic and Evolutionary computing techniques are also employed [12, 13].

The OCR method for character extraction consists of the following five steps:

1. Image acquisition

2. Pre-processing

3. Segmentation

4. Normalization

5. Template matching

Each of these steps is a field unto itself, performed by proper OCR - Code. The OCR program should perform the background noise elimination in the image input and feature extraction to detect the characters in the image.

Image Acquisition: In image processing, the major step is image acquisition. This step has been just described in section 2.1. Image acquisition is to capture the target image, normally with a digital camera. Image captured signal from camera (analog) and data (image file) need to be identified and converted to digital form. Image captured in digital format can be easily processed by computers. The image quality should be good for good accuracy in OCR, so a camera with high resolution is used.

Pre-Processing: Pre-processing is the operation on the image to be uploaded into OCR program. The image is processed by noise smoothing and filtering. The scanned image is converted from RGB (Red Green Blue) to grayscale or to its binary picture.

Segmentation: Segmentation may be performed at two levels. On the first level, text, graphics and other parts are separated. On the second level, text lines, words and characters in the image are located [14]. Each and every character of the text in the input image is divided as shown in Fig.5, by assigning correct character size using labeling operation. Segmentation further performs character resizing into a pixel array. Based on a threshold value for brightness, each pixel of the greyscale image is differentiated as either dark or bright, column by column and row by row.

Normalization: The resulting image from segmentation is processed to normalize the character size to $42 * 24$ pixels, which is the size of the template image, for fast matching of the image characters. Template image of a character after pixel (size) normalization is shown in Fig.6. 
Template matching procedure: OCR is exercised through a program that can recognize characters by the template matching. Template matching technique works by comparing the segmented and normalized characters with that of each and every alphabetical letter (A-Z) and number (0-9), available as standard bitmaps of size $42 * 24$, as shown in Fig.7. A total of 36 alphanumeric characters are thus checked for a match, pixel by pixel, for every character of the string. For matching images, we can elucidate the character from its bitmap, with logic " 1 " and " 0 " for the pixel value representing the character against its background respectively. Template matching process includes the following steps.

a. A string of the character to be extracted should be given as an image input initially to the main program for OCR.

b. After segmentation, the image should be rescaled to $42 * 24$ bitmap character size and we get a series of images corresponding to the length of the string.

c. Rescaled image is compared/matched with the template bitmap images (also of $42 * 24$ size) based on a correlation function.

d. Each of the rescaled character images that is highly matching with template image will be stored.

e. If the match is not found, these steps should be repeated after another noise filtering stage.

f. Text output with the highest match will give correct character output.

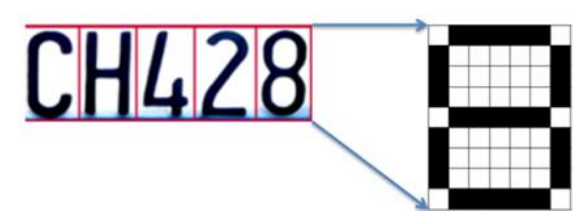

Fig.5. Segmented Characters from Input Image

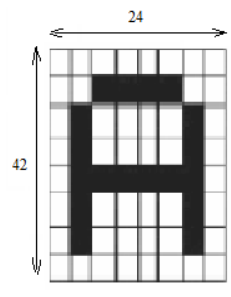

Fig.6. Character Templates with Pixels Normalization

\begin{tabular}{|c|c|c|c|c|c|c|c|c|c|}
\hline i & 2 & 3 & 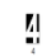 & 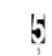 & & 6 & II & 8 & \\
\hline 0 & i) & : & : & 6 & & & II & I & \\
\hline$I$ & 0 & d: & 0 & i & & & II & I & \\
\hline & 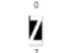 & & & & & & & & \\
\hline
\end{tabular}

Fig.7. Template Character Fonts Stored in OCR Program.

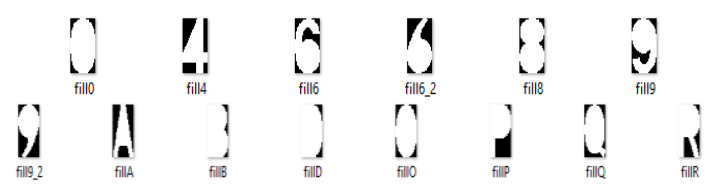

Fig.8. Templates with Different Fill Area Background Change 
Correlation calculations are done for the normalized characters by comparing its pixel array with that of the templates. By calculating correlation for each and every character of the string in sequence, the OCR program will give the extracted output.

In case of any errors in identification, the character image to be recognized is preferably dilated and uploaded again to the OCR program. The threshold value (pixel value that differentiates the dark and the bright pixel of the image uploaded) may be changed and the OCR process repeated to reduce the recognition error. Sometimes, error may also be seen in the form of a near match out like $\mathrm{O}$ as 0 , D as $\mathrm{O}, \mathrm{A}$ as 4 and $\mathrm{S}$ as 5 . In case the template is not matching with the right character, it may be required to include additional templates of varying fronts along with standard tempate images. Such fonts, for example, may have unfilled background color (white) area and filled background color (black) area, as indicated in the Fig. 8.

RAMSHANKAR

YARLAGADDA

Fig.9. Text image to be Scanned

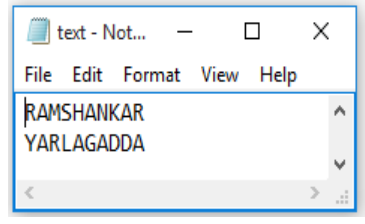

Fig.10. Recognized Text.

MATLAB Image processing Toolbox: MATLAB image processing functions can identify the image of the set of alphabets and numbers. The Normalized correlation function is used to match the input letters with existing standard thirty-six templates. The size should be greater than or equal to $42 * 24$ pixels. The OCR program developed in MATLAB has been used for a test case of alphabetic characters and the test sample is shown in Fig.9, while the text recognized by OCR system is shown in Fig.10.

\section{Design and Development of Pan Tilt Camera}

\subsection{Camera selection}

Camera selection is mainly based on its cost, ease of handling and quality of image produced. Colour and grey scale CCD camera could be used to acquire images of engraved contours in an industrial environment [10]. Reliable results have also been reported using web camera [15-17]. A digital camera (Sony DCR SX21) is proposed for VIN inspection, which is having good resolution of $0.56 \mathrm{MP}$ and autofocus. The digital camera is interfaced to a computer usinng USB data cable connection.

\subsection{Camera fixture}

The mechanical design of the camera fixture, consisting of a conveyor belt wrapped over two wheels fixed on a base plate, is shown in the Fig. 11 and 12. The pan-tilt camera frame is fabricated using Poly Propylene sheet and holds the camera. It is tied to the plastic conveyor belt and helps to adjust the angular position of camera. It makes use of a gear mechanism made up of a gear mesh, at the bottom center of the frame, to turn 
the camera frame.

Main body: The rectangular main body is made of plywood and forms a base, onto which, the conveyor wheels and two guide rods are fixed, as shown in Fig. 11 and Fig.12. The guide rods remain $13 \mathrm{~cm}$ apart giving sufficient space for the conveyor mechanism in between. The guide rods bear the weight of the camera frame while allowing it to slide along its length, in tandem with the conveyor belt. Two aluminum linear ball bearing blocks are used to smoothly slide the camera fixture (seated on the conveyor) along the rods. The two conveyor wheels are used to move the conveyor forward or backward. One of the wheels is driven by stepper motor(1). The other wheel is mounted on a circular shaft, freely supported by a bearing. Belt conveyor has enough grip on the wheels, for effective transmission of motion. The electrical control circuit is fitted beside the belt drive.

Pan-tilt arrangement for camera frame: Pan-tilt camera frame, shown in Fig.13, is designed to mount the camera at height of $45 \mathrm{~cm}$ from the base (corresponding to VIN location on chassis) and to turn or tilt the camera to a certain angle $\left(0^{\circ}-360^{\circ}\right)$ required for capturing an image of VIN sticker located on the center pillar at the car door. The camera frame is made in two parts. The upper part holds the camera and is connected through a gear mesh to the lower part having the stepper motor(2) drive. The lower part rests and slides on the two steel rods ( through linear bearings) and is also tied onto the conveyor belt, as mentioned before. When the conveyor belt moves, it effectively moves the camera frame.

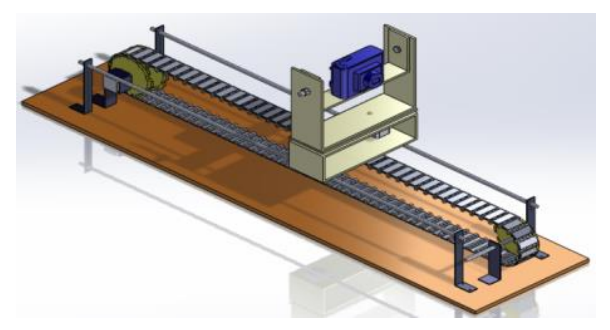

Fig.11. Machine vision System (Isometric view)

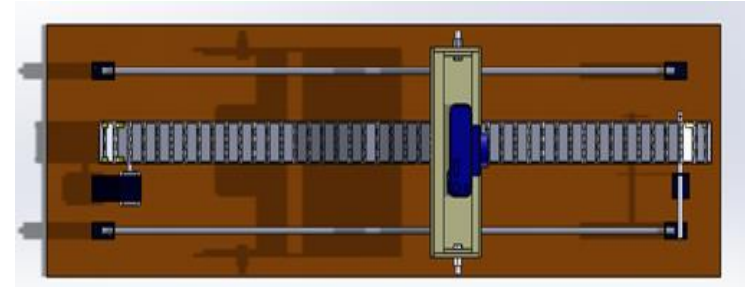

Fig.12. Top view of Camera Fixture

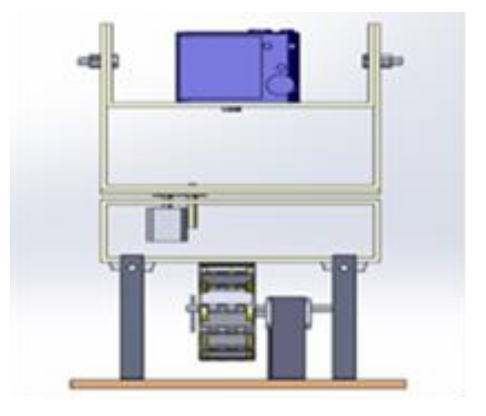

Fig.13. Side view of Camera Fixture 
Joystick control: Stepper motor(1) is controlled automatically or with a joystick in horizontal direction. At the same time, stepper motor(2) for camera tilt, is controlled automatically or with joystick. In the case of any positioning error of the pan-tilt camera system in locating the VIN for image capturing, the joystick helps in controlling two stepper motors about $\mathrm{X}$ and $\mathrm{Y}$ axes to get fine adjustment.

Electronic control of stepper motors: The camera fixture just described utilizes two NEMA 17 Stepper motors of $12 \mathrm{~V}$ rating and a conveyor meachanism with carrying capacity of $4.2 \mathrm{~kg}$ for camera movement. NEMA 17 stepper motors (4kgm Torque), coupled with Easy stepper drive, provide very precise control, for example, in CNC conveyors and other material handling applications. A step-down transformer ( $230 \mathrm{~V}$ to $12 \mathrm{~V}$ AC; 2 A power supply) is used to run the two stepper motors using 'Easy stepper motor drives (V44A3967)'. Rotation of camera frame about vertical axis and translation along horizontal axis are controlled by the electronic control system. Stepper motor direction is controlled by the arduino programming software with a fixed pulse and step angle $\left( \pm 1.8^{\circ}\right)$ of rotation. Arduino UNO R3 is provided with the $5 \mathrm{~V}$ power supply to run the total control unit and joystick.

Arduino IDE: Arduino board consists of an ATMEGA328 microcontroller and an IDE that runs on a PC computer, to write and upload computer code to the board. The Arduino UNO is used to control all the required operations of the vision system considering all the parameters. Arduino offers lots of advantages over other microcontrollers as it is inexpensive, gives clear programming environment, open source and extensible hardware. Figure 14 shows the electronic control approach for the vision system and Fig. 15 shows the schematic circuit diagram of Arduino based stepper motor control.

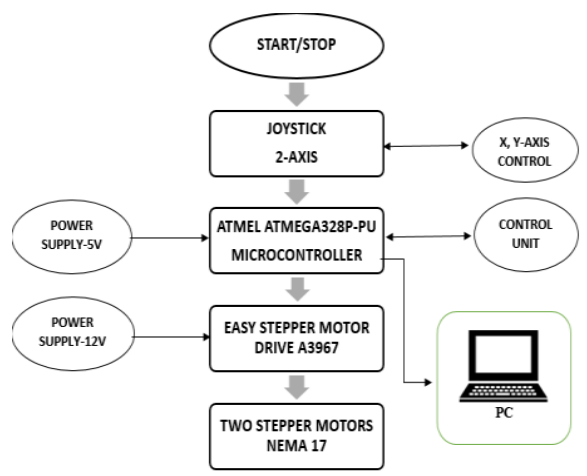

Fig.14. Block Diagram of Machine vision System

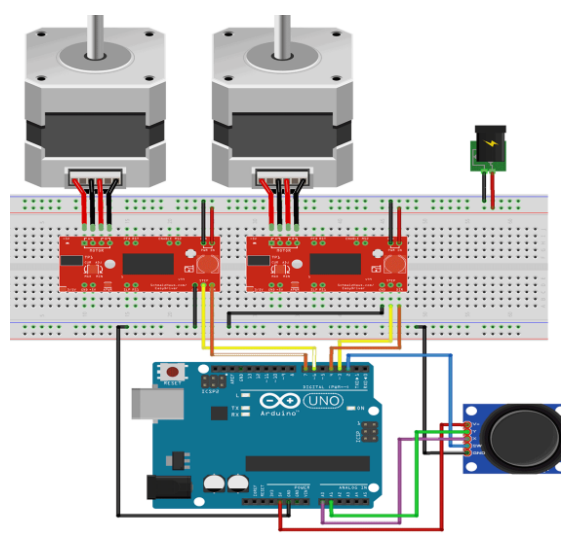

Fig.15. Schematic Circuit Diagram of Arduino based Stepper Motor Control. 


\section{MATLAB based GUI}

\subsection{The design of MATLAB Graphic User Interface}

A GUI is created by designing the layout of 'user interface window' and writing 'callback functions' that perform different operations when the user clicks the relevant features in user interface window [18]. Graphical User Interface Layout Toolbox in MATLAB provides various types of features, like Select, Pushbutton, Checkbox, Edit text, Toggle button, Text fields, Slides, Axes, etc. Blank 'guide' template layout enables the layout structure of GUI components as per our requirement. It enhances the user interaction with OCR program.

There are mainly three elements in creating GUI, namely, callbacks, figures and components.

Call back: Code performed in response to an event is known as a callback. MATLAB should be able to respond to each and every event, such as key pressed.

Figures: Components of GUI must be arranged within the window on the PC screen. Axes tool is used to locate the figure position in GUI.

Components: Components in MATLAB GUI provide different controls like a push button, edit boxes, axes, slides, menus, etc.

GUI layout Toolbox of MATLAB can automatically generate M-file, which can be used to control the GUI functions. The M-file initializes the GUI and contains a frame for the commonly used callbacks for each component. M-files can be edited in M-file editor and can add callback codes for executing the image processing and template matching functions [19].

Figure 16 is a screenshots of GUI Homepage. The user interface home page displays the required options for uploading the VIN image manually. Clicking the white button on the right side allows the user to upload the image. Loaded VIN image is displayed on home page screen. Green color button should be clicked by the user for processing image. For best results the VIN image is loaded after noise smoothing or filtering. Figure 17 shows the user interface window with the VIN image uploaded.

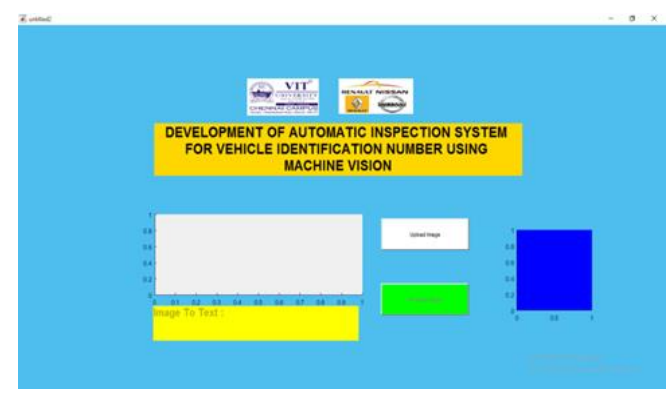

Fig.16. GUI Home Page

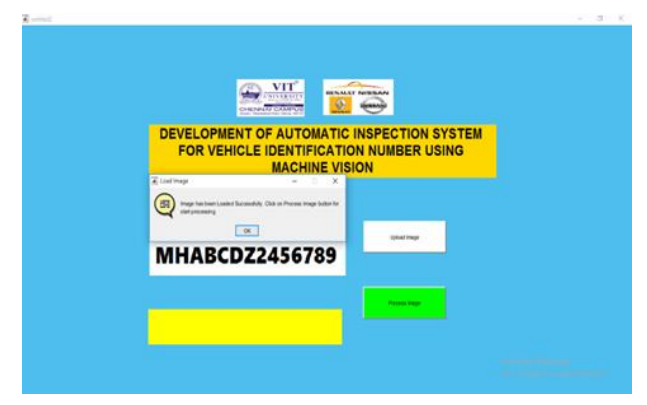

Fig.17. VIN Image Uploaded 


\section{System Performance Issues in Implementation}

The various activities involved in the automatic inspection of VIN using an off-line OCR system can be summarized in sequence as follows. With the help of image capture devices, the image of VIN sticker is captured and cropped to the size of VIN string. The VIN image is subject to noise smoothing and uploaded to the OCR program (MATLAB Toolbox) through its Graphic User Interface (GUI). The OCR processes the image and stores the digital VIN string of characters in a file and also displays it through a GUI. Notable issues in the performance of this vision system are,

- The size of template should not be less than $42 * 42$ pixels

- Input picture (or) image can be any colored also.

- If the character in the image is thin (or) unable to be detected, the image correction is performed to increase the thickness.

- Time taken for the image processing by OCR program is 3 to 4 seconds. Processing time on PC with more RAM will be less.

- More processing time can be reduced by using image template size of $20 * 20$ pixels.

While the image is processed, the template matching trial with thirty-six characters can be seen on the display beside the buttons. These characters have been loaded in the same folder to facilitate callback of the respective template for character recognition. Finally, it shows the template that gives an exact match.

Results of OCR for a sample VIN image is shown in a separate yellow box of the user interface window, shown in Fig. 18. All the fourteen characters in the printed VIN image are extracted and displayed as a text string (text read from the image) in the yellow box as shown. The OCR accuracy is $100 \%$ with all alphabetic and numerical characters.

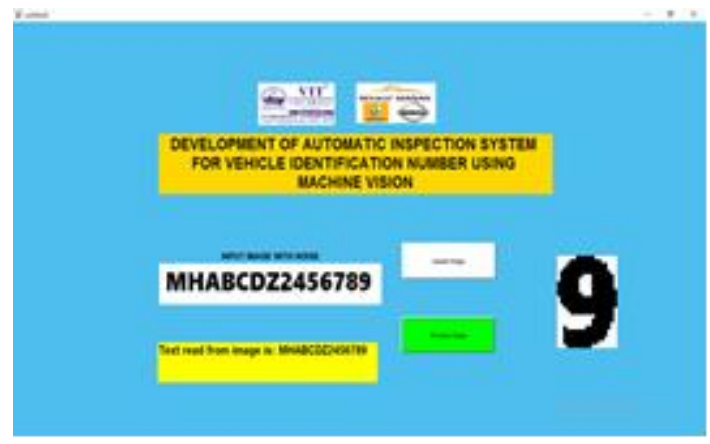

Fig.18. Screenshot of VIN Extracted from Its Image

\section{Conclusion}

Machine vision is a convenient technique for the inspection of VIN of vehicles. The MATLAB based OCR system is mainly developed to automatically extract the VIN character string, from the photo image of the same.

- A technique has been proposed for the automatic capture of VIN image, from a sticker placed at the car door. For this, a movable camera fixture has been fabricated and tested.

- The major operations in this automatic VIN inspection system include, capturing VIN image, OCR processing of pre-processed VIN image and display of the extracted VIN string through GUI. These operations are successfully carried out using MATLAB. 
- Techniques to overcome errors probable in OCR implementation, like font variations, thresholding, etc., are discussed. In case the database of character templates is not matched with exact character, it is required to add more templates with varying fronts into the database.

- Implementation of vision system can reduce human effort, inspection time and improved quality in the inspection line.

\section{References}

[1] Bhat R, Mehandia B. Recognition of vehicle number plate using matlab. International Journal of Innovative Research in Electrical, Electronics, Instrumentation and Control Engineering. 2014; 2(8): 1899-1903.

[2] Ahmed AU, Masum TM, Rahman MM. Design of an automated secure garage system using license plate recognition technique. International Journal of Intelligent Systems and Applications. 2014; 6(2): $22-28$.

[3] Renukadevi. D, Kanagapushpavalli. D. Automatic license plate recognition. Trendz in Information Sciences and Computing (TISC), 3rd IEEE International Conference. 2011; 75-78.

[4] Khan JA, Shah MA. Car Number Plate Recognition (CNPR) system using multiple template matching. Automation and Computing (ICAC), 22nd International Conference. 2016; 290-295. IEEE.

[5] Gupta P, Purohit GN, Rathore M. Number Plate extraction using Template matching technique. International Journal of Computer Applications. 2014; 88(3): 40-44.

[6] Shah P, Karamchandani S, Nadkar T, Gulechha N, Koli K, Lad K. OCR-based chassis-number recognition using artificial neural networks. In Vehicular Electronics and Safety (ICVES), 2009 IEEE International Conference. 2009; 31-34.

[7] Yuanyuan Z. Research on automatic visual inspection method for character on cartridge fuse based on template matching. In Information Science and Control Engineering (ICISCE), 2016 3rd International Conference. 2016; 527-531. IEEE.

[8] Majumder A. Image processing algorithms for improved character recognition and components inspection. In Nature \& Biologically Inspired Computing (NaBIC 2009), World Congress. 2009; 531536. IEEE.

[9] Luo B, Guo G. Fast printing defects inspection based on multi-matching. In Natural Computation, Fuzzy Systems and Knowledge Discovery (ICNC-FSKD), 12th International Conference. 2016; 1492-1496. IEEE.

[10] Ahmad NB, Sulaiman MB, Aripin MK. Quality inspection of engraved image using shape-based matching approach. In Mechatronics (ICOM), 4th International Conference. 2011; 1-6. IEEE.

[11] Farhat A, Al-Zawqari A, Al-Qahtani A, Hommos O, Bensaali F, Amira A, Zhai X. OCR based feature extraction and template matching algorithms for Qatari number plate. In Industrial Informatics and Computer Systems (CIICS), International Conference. 2016; 1-5. IEEE.

[12] Chaudhuri A, Mandaviya K, Badelia P, Ghosh SK. Optical character recognition systems for different languages with soft computing. Springer International Publishing; 2017.

[13] Katiyar G, Mehfuz S. A hybrid recognition system for off-line handwritten characters. Springer Plus; 2016; 5(1): 357.

[14] Manoj TH, Rubia AS. Text recognition in street level images. International Journal of Emerging Technology and Advanced Engineering. 2013; 3: 392-395.

[15] Souza LR, Oliveira RM, Stoppa MH. Proposal of Automated Inspection Using Camera in Process of VIN Validation. InMultibody Mechatronic Systems, Springer, Cham; 2015; 285-293.

[16] Shah AN, Gaikwad AS. A review - Recognition of license number plate using character segmentation and OCR with template matching, International Journal of Advanced Research in Computer and Communication Engineering. 2016; 5: 159-162. 
[17] Al-Ghaili AM, Mashohor S, Ramli AR, Ismail A. Vertical-edge-based car-license-plate detection method. IEEE Transactions on Vehicular technology. 2013; 62(1): 26-38.

[18] Hunt BR, Lipsman RL, Rosenberg JM. A guide to MATLAB: for beginners and experienced users. Cambridge University Press; 2014.

[19] Mao Q, Pietrzko S. Control of noise and structural vibration. London: Springer; 2013.

\section{Authors' Profiles}

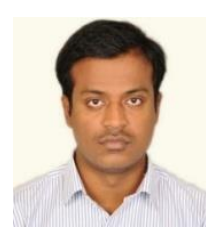

Yarlagadda Ramshankar, completed B.E in Mechanical Engineering from IFHE, Hyderabad (2014) and MTech (Mechatronics) from VIT University, Chennai (2017).

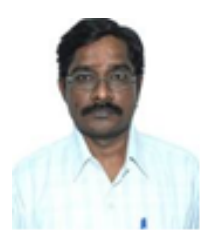

Dr.Deivanathan.R, PhD in Mechanical Engineering from IIT, Madras (2005), is presently working as Associate Professor at VIT University, Chennai.

How to cite this paper: Yarlagadda Ramshankar, Deivanathan R,"Development of Machine Vision System for Automatic Inspection of Vehicle Identification Number", International Journal of Engineering and Manufacturing(IJEM), Vol.8, No.2, pp.21-32, 2018.DOI: 10.5815/ijem.2018.02.03 